\title{
The role of polyethylene wax on the thermal conductivity of
}

transparent ultradrawn polyethylene films

Xinglong Pan,,$^{\dagger}$ Albert H. P. J. Schenning, ${ }^{\dagger, \dagger}$ Lihua Shen, ${ }^{\dagger, \S}$ and Cees W. M. Bastiaansen ${ }^{\dagger, \|, *}$

${ }^{\dagger}$ Laboratory of Stimuli-Responsive Functional Materials \& Devices, Department of Chemical Engineering and Chemistry, Eindhoven University of Technology, Den Dolech 2, 5612 AZ, Eindhoven, The Netherlands

Institute for Complex Molecular Systems, Eindhoven University of Technology, Den Dolech 2, 5612 AZ, Eindhoven, The Netherlands

$\S$ Department of Mechanical Engineering, University of Colorado Boulder, Colorado, Boulder CO 80309, U.S.A.

${ }^{\mid}$School of Engineering and Materials Science, Queen Mary, University of London, London E1 4NS, United Kingdom

Email address: C.W.M.Bastiaansen@tue.nl (C. W. M. Bastiaansen) 

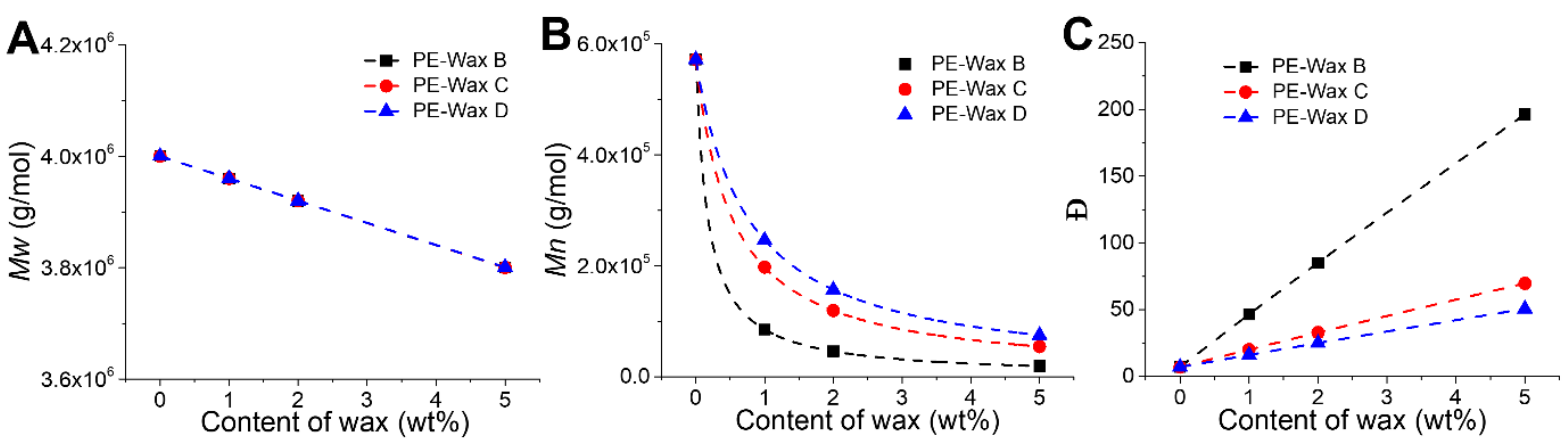

Figure S1 (A) Mw, (B) Mn and (C) PDI (尹) of pure PE and PE-wax films.

Mw, Mn, and PDI were calculated by the following equations:

$$
\begin{aligned}
& M w_{f}=M w_{w} \varphi+M w_{p}(1-\varphi) \\
& \frac{1}{M n_{f}}=\frac{\varphi}{M n_{w}}+\frac{(1-\varphi)}{M n_{p}} \\
& \text { PDI }=\frac{M_{w}}{M_{n}}
\end{aligned}
$$

Here, $\mathrm{Mw}_{\mathrm{f}}, \mathrm{Mw}_{\mathrm{w}}$, and $\mathrm{Mw}_{\mathrm{p}}$ are the $\mathrm{Mw}$ of films, wax, and polyethylene. $\mathrm{Mn}_{\mathrm{f}}, \mathrm{Mn}_{\mathrm{w}}$, and $\mathrm{Mn}_{\mathrm{p}}$ are Mn of films, wax, and polyethylene. $\varphi$ is the content of wax to film. 

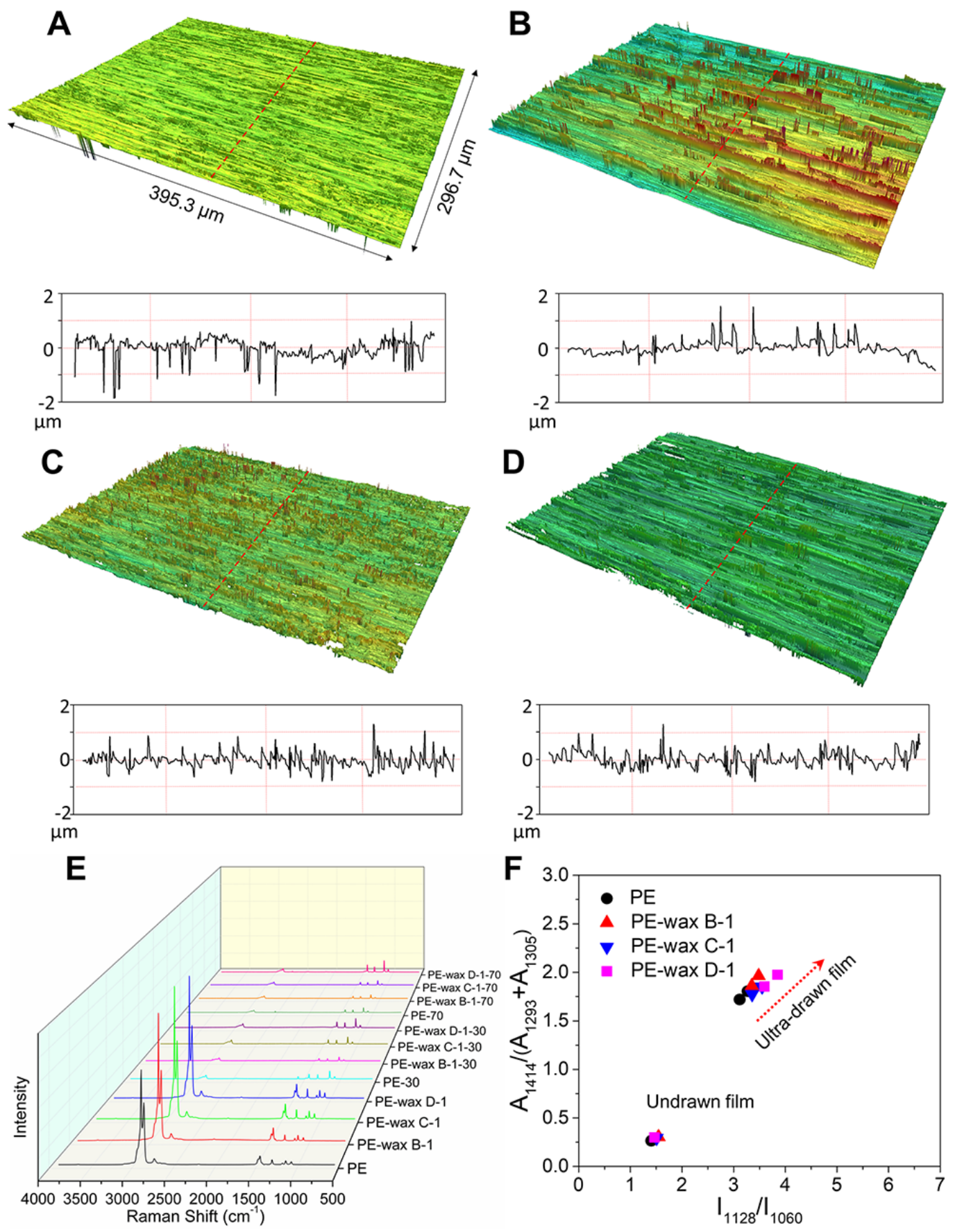

Figure S2 Surface (top) structure and roughness of (A) PE-30, (B) PE-wax B-1-30, (C) PEwax C-1-30 and (D) PE-wax D-1-30 films. The scanning follows the red line. (E) Raman spectra of PE and PE-wax film with draw ratios of 1, 30 and 70, (F) Ratio of integral areas of Raman band at $1414 \mathrm{~cm}^{-1}$ to Raman bands at $1293 \mathrm{~cm}^{-1}$ plus $1305 \mathrm{~cm}^{-1}$ vs. intensity ratio of Raman bands at $1128 \mathrm{~cm}^{-1}$ and $1060 \mathrm{~cm}^{-1}$. 


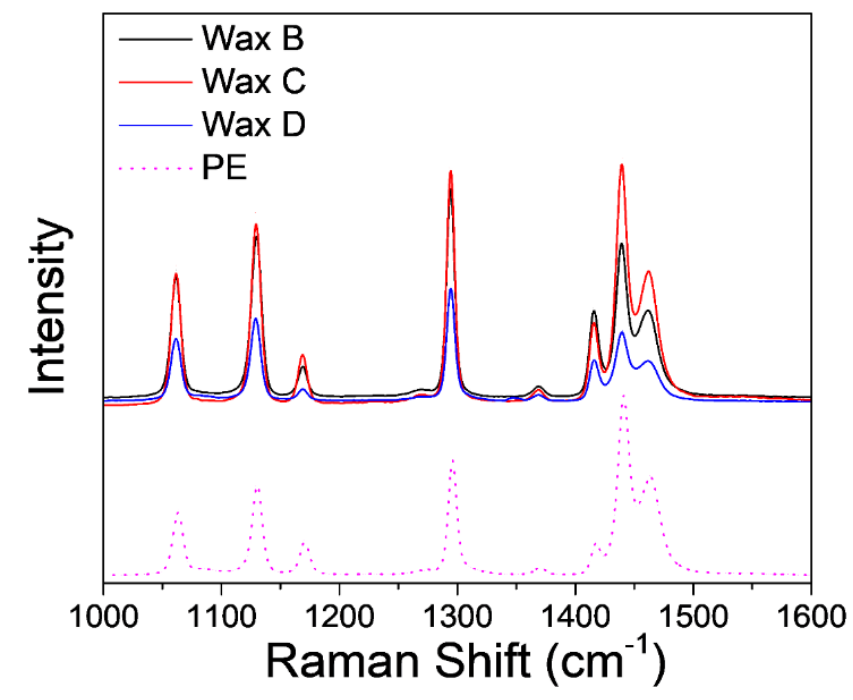

Figure S3 Raman spectra of wax B, wax C, wax D, and PE powder.

Table S1 Crystallinity of samples measured by DSC

\begin{tabular}{c|c|c|c}
\hline & undrawn & 30 times & 70 times \\
\hline PE & $\sim 71 \%$ & $\sim 80 \%$ & $\sim 84 \%$ \\
\hline PE-wax B-1 & $\sim 73 \%$ & $\sim 78 \%$ & $\sim 84 \%$ \\
\hline PE-wax C-1 & $\sim 72 \%$ & $\sim 81 \%$ & $\sim 86 \%$ \\
\hline PE-wax D-1 & $\sim 72 \%$ & $\sim 82 \%$ & $\sim 88 \%$ \\
\hline
\end{tabular}


Table S2 $<\cos ^{2} \beta>$ and orientation function (f) of samples with a draw ratio of 30

\begin{tabular}{l|l|l}
\hline Samples & $\left\langle\cos ^{2} \boldsymbol{\beta}\right\rangle$ & $\mathbf{f}$ \\
\hline \multirow{2}{*}{ PE-wax B 1-30 } & 0.904 & 0.857 \\
\cline { 2 - 3 } & 0.914 & 0.872 \\
\hline \multirow{2}{*}{ PE-wax C 1-30 } & 0.929315 & 0.894 \\
\cline { 2 - 3 } PE-wax D 1-30 & 0.883 & 0.825 \\
\hline
\end{tabular}

The orientation function (f) of samples with a draw ratio of 30 was calculated based on the intensity of two scattering dots. All values of $\mathrm{f}$ calculated based on the peak width at the half peak height of the peak at $<200>$. 

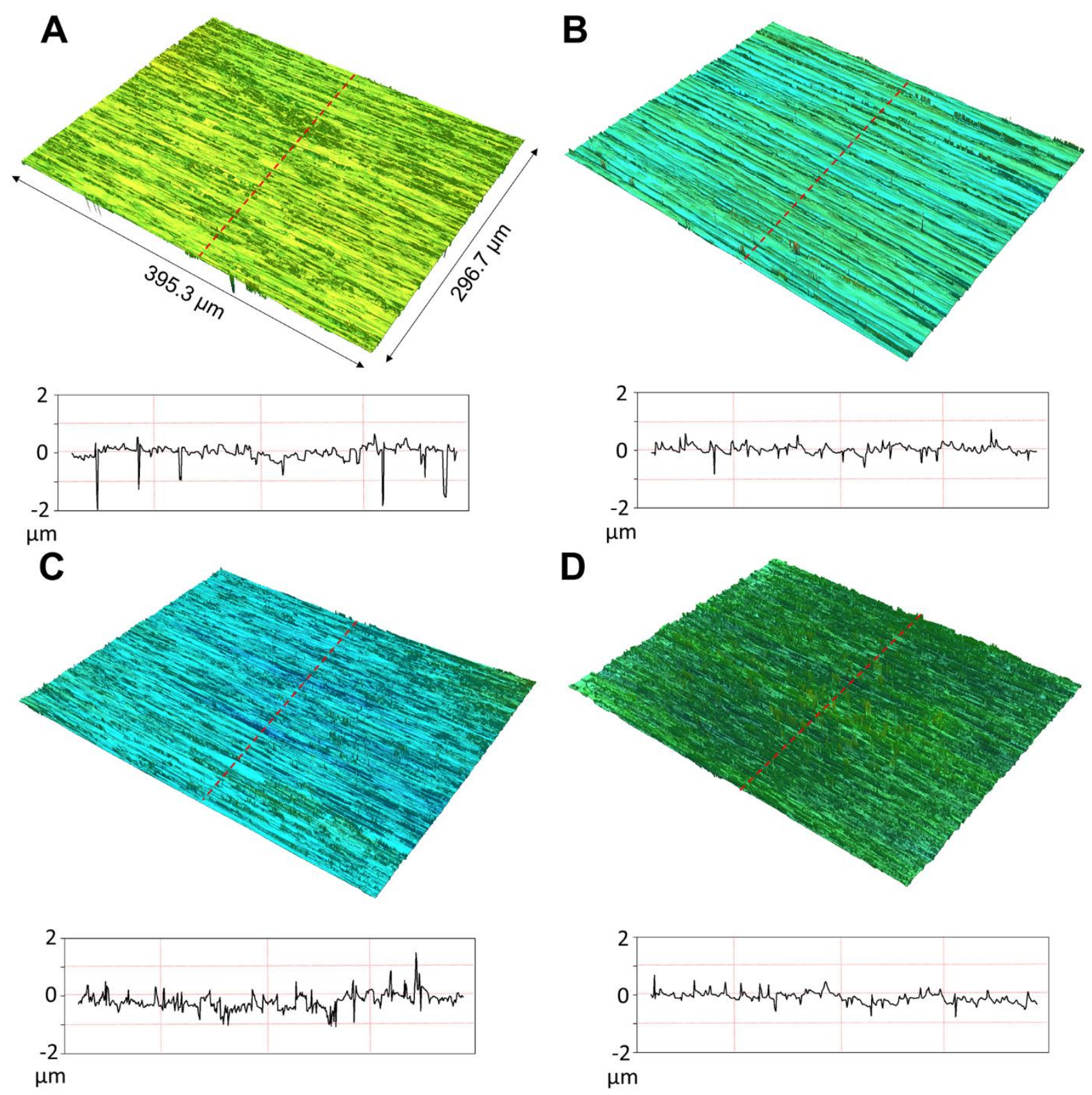

Figure S4 Surface (bottom) structure and roughness of (a) PE-30, (b) PE-wax B-1-30, (c) PEwax C-1-30 and (d) PE-wax D-1-30 films. 


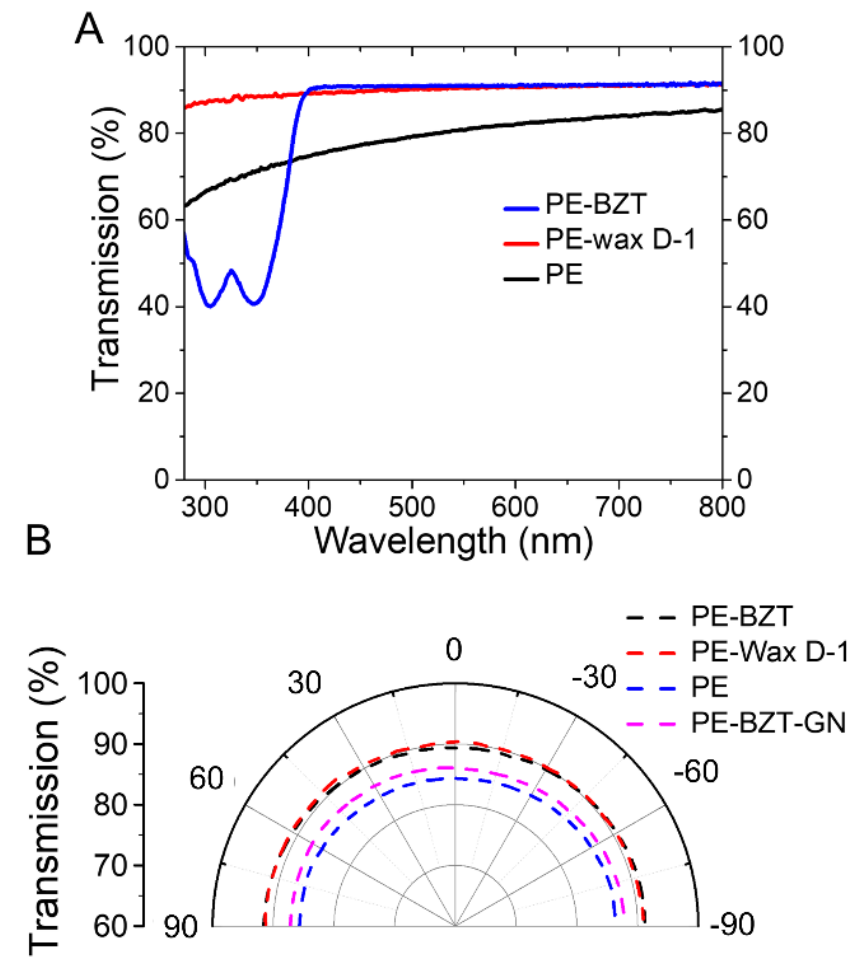

Figure S5 A) UV-vis spectra and B) polarized visible light transmission of PE, PE-wax D-1, PE-BZT-30 and PE-BZT-GN(graphene) films (The angular axes in polarized spectra represents the angle between the drawing direction of samples and the direction of polarizer). ${ }^{1}$

$\mathrm{S}$, defined as the ratio of the transmission difference between parallel and perpendicular direction with the perpendicular transmission $\left(\left(T_{\text {parallel }}-T_{\text {perpendicular }}\right) / T_{\text {perpendicular }}=\Delta T / T_{\text {perpendicular }}\right)$, for PE, PE-BZT, PE-BZT-GN, and PE-wax D films are about 0.020, 0.024, 0.020, and 0.011, respectively. The refractive index of BZT is approximately 1.575 while the ordinary and extraordinary refractive indexes in drawn PE are approximately 1.57 and 1.52 at 0 and 90 degrees. Therefore, the difference of visible light transmission of PE-BZT films at 0 and 90 degrees might be attributed to the mismatched refractive indexes between pure BZT, BZT/graphene and $\mathrm{PE}$ in the perpendicular of drawing direction. 

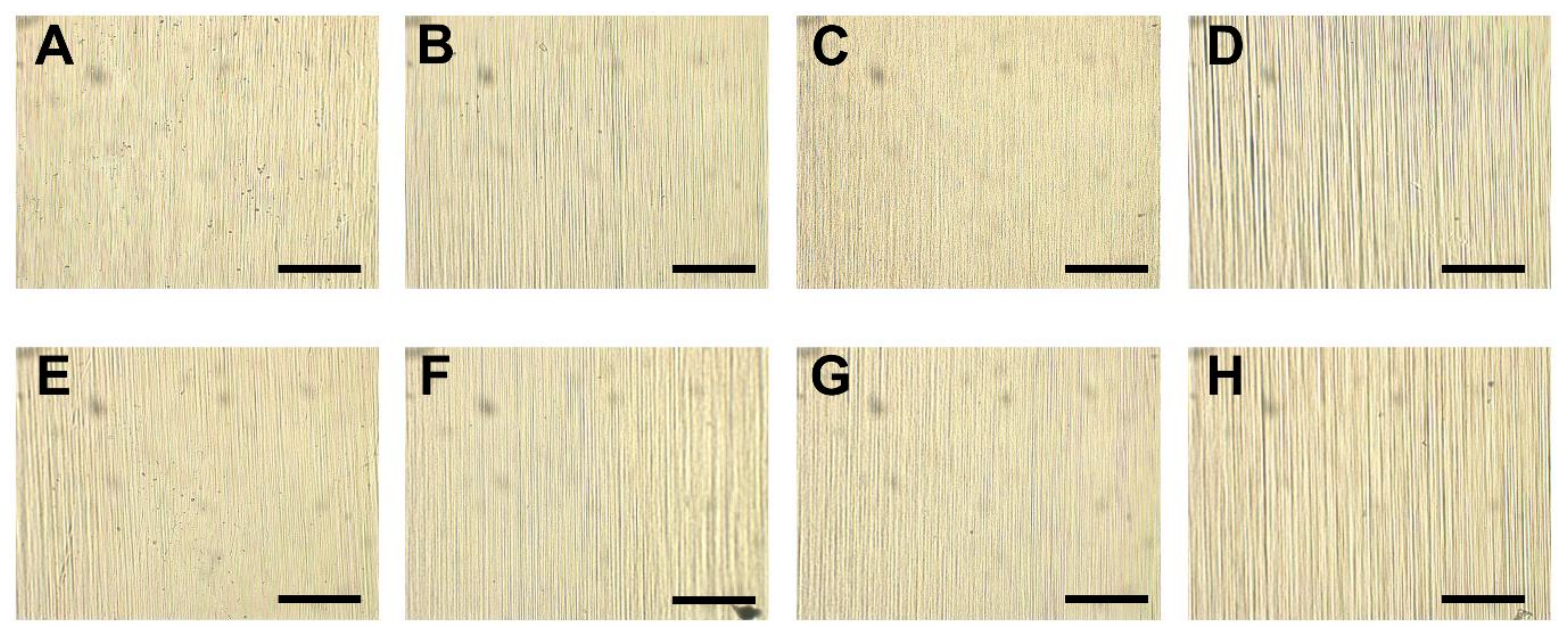

Figure S6 Optical microscopy images of (a) PE-30, (b) PE-wax B-1-30, (c) PE-wax C-1-30, (d) PE-wax D-1-30, (e) PE-70, (f) PE-wax B-1-70, (g) PE-wax C-1-70 and (h) PE-wax D-1-70 films. Scale: $100 \mu \mathrm{m}$. 

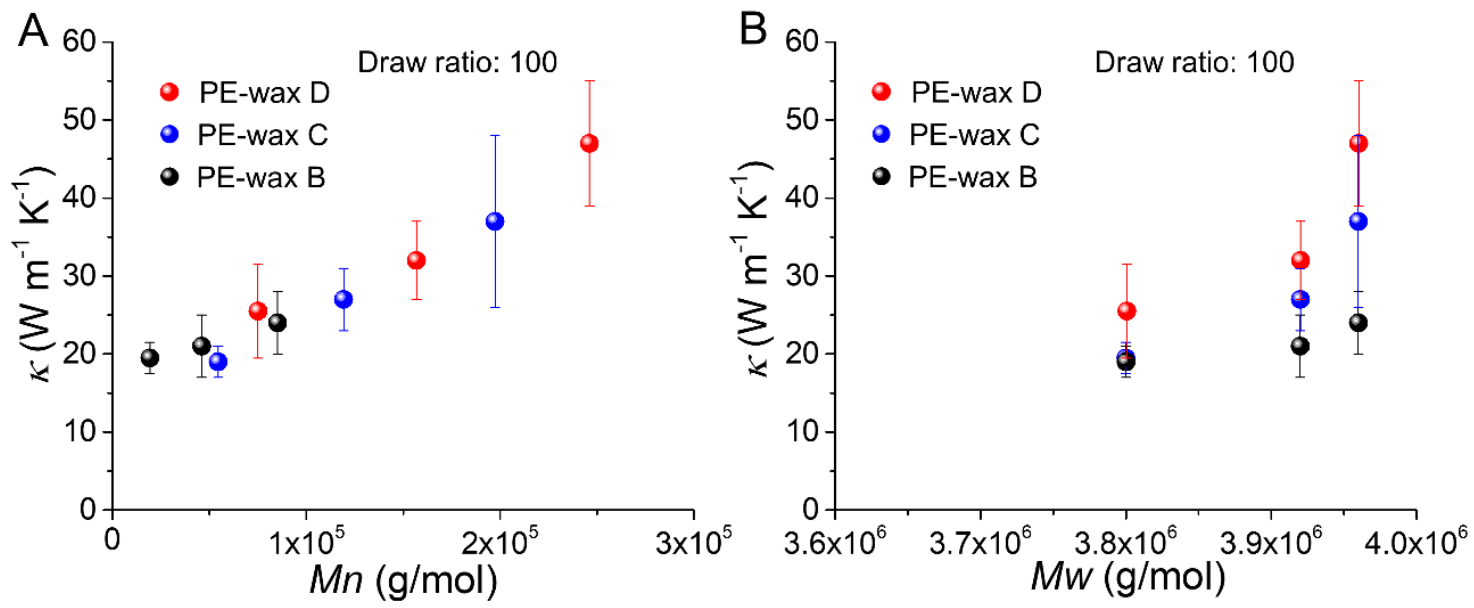

Figure S7 Thermal conductivity of PE-wax films with a draw ratio of 100 as functions of Mn and Mw.

Table S3 Fitting values $\left(\mathrm{R}^{2}\right), \kappa_{1}$, and $\kappa_{2}$

\begin{tabular}{c|c|c|c}
\hline Reference & $\mathbf{R}^{\mathbf{2}}$ & $\boldsymbol{\kappa}_{\mathbf{1}}$ & $\kappa_{\mathbf{2}}$ \\
\hline PE-wax B-1 & 0.971 & $25.1 \pm 4.35$ & $1.168 \pm 0.140$ \\
\hline PE-wax C-1 & 0.814 & $33.8 \pm 5.49$ & $0.772 \pm 0.257$ \\
\hline PE-wax D-1 & 0.957 & $47.5 \pm 7.35$ & $0.992 \pm 0.165$ \\
\hline
\end{tabular}


Table S4 Ultimate thermal conductivity calculated by equation 1 based on experimental data ( $\kappa)$ and predicted thermal conductivity by equation $4(\kappa)$

\begin{tabular}{c|c|c}
\hline Reference & $\kappa$ & $\kappa$ \\
\hline PE-wax B-1 & $25 \pm 4$ & 25.83 \\
\hline PE-wax C-1 & $34 \pm 5$ & 36.95 \\
\hline PE-wax D-1 & $47 \pm 7$ & 40.60 \\
\hline PE & $43.2 \pm 4$ & 58 \\
\hline PE 2-4 & $47.5 \pm 2.16$ & 47.6 \\
\hline PE 6-7 & $52.6 \pm 1.84$ & 71.8 \\
\hline PE10-7 & $60.1 \pm 4.61$ & 88 \\
\hline
\end{tabular}

The experimental value could be a little lower than the predicted value based on this theory due to the effects of chain entangle, the side chain and defects inside of films, which were not considered in our work. 


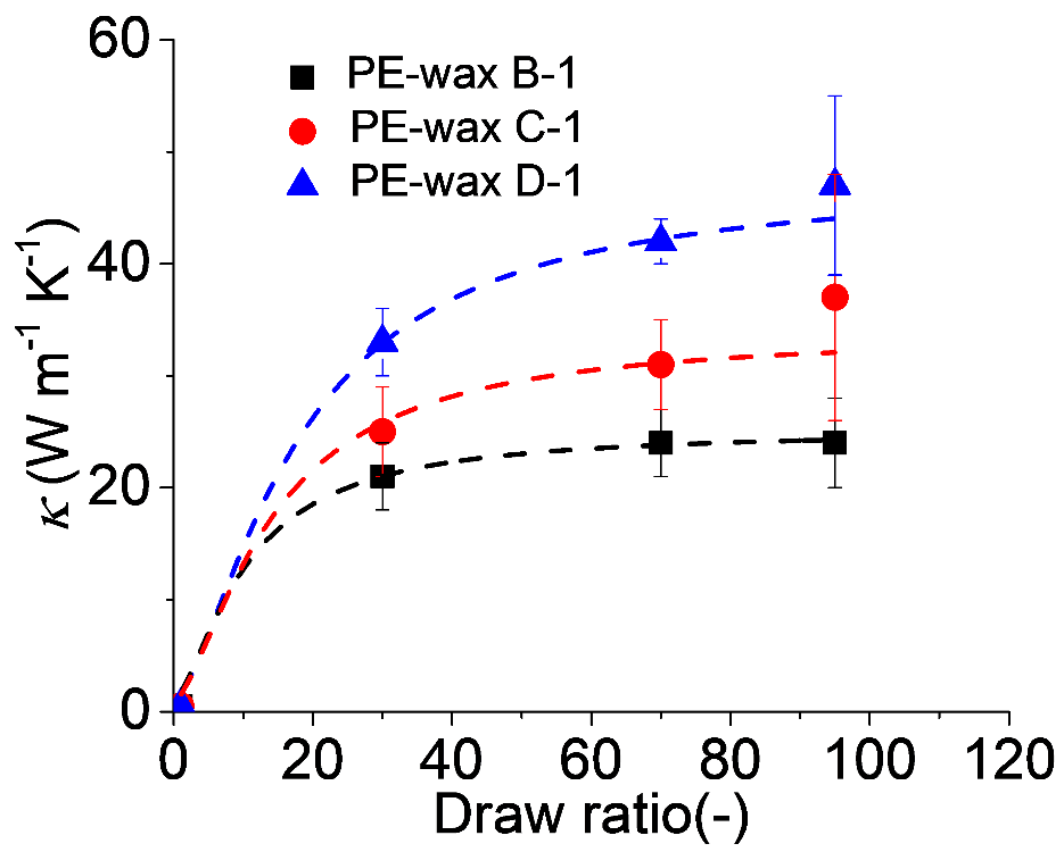

Figure S8 Thermal conductivity of ultradrawn PE-wax films. The dashed lines are the fitting curves based on equation 3 .

\section{Model}

A few remarks are appropriate with respect to the model presented in the main text. Firstly, the model (and experimental data) indicate that the thermal conductivity of ultra-drawn systems is governed by the draw ratio and number average molecular weight. The model is based on the pseudo-affine deformation scheme, it assumes an isotropic starting morphology and that defects such as grain boundaries are absent. As a consequence, large deviations from the model presented here are expected, for instance, fibre spinning operations and/or continuous cast film extrusion lines due to pre-orientation effects. ${ }^{2,3}$ Secondly, the structure and properties of (commercial) ultra-drawn UHMW-PE films depend rather strongly on their processing. For instance, additional defect structures related to partially preserved powder morphologies in "virgin or nascent" systems might be a source of deviations from our model. ${ }^{4}$ 
(1) Pan, X.; Shen, L.; Schenning, A. P. H. J.; Bastiaansen, C. W. M. Transparent, HighThermal-Conductivity Ultradrawn Polyethylene/Graphene Nanocomposite Films. Adv Mater 2019, 1904348.

(2) Shen, S.; Henry, A.; Tong, J.; Zheng, R.; Chen, G. Polyethylene Nanofibres with Very High Thermal Conductivities. Nat Nanotechnol 2010, 5, 251-255.

(3) Xu, Y.; Kraemer, D.; Song, B.; Jiang, Z.; Zhou, J.; Loomis, J.; Wang, J.; Li, M.; Ghasemi, H.; Huang, X.; et al. Nanostructured Polymer Films with Metal-like Thermal Conductivity. Nat Commun 2019, 10, 1771.

(4) Ronca, S.; Igarashi, T.; Forte, G.; Rastogi, S. Metallic-like Thermal Conductivity in a Lightweight Insulator: Solid-State Processed Ultra High Molecular Weight Polyethylene Tapes and Films. Polym 2017, 123, 203-210. 